\title{
Evaluation of the humoral and cellular immune response to different antigens of Corynebacterium pseudotuberculosis in Canindé goats and their potential protection against caseous lymphadenitis
}

\author{
L.F. Moura-Costa *, R.C. Bahia, R. Carminati, V.L.C. Vale, B.J.A. Paule, \\ R.W. Portela, S.M. Freire, I. Nascimento, R. Schaer, L.M.S. Barreto, R. Meyer \\ Departamento de Biointeração - Instituto de Ciências da Saúde - Universidade Federal da Bahia, \\ Av. Reitor Miguel Calmon, S/N - Vale do Canela, CEP 40140-100, Salvador-BA, Brazil
}

Received 6 December 2007; received in revised form 6 May 2008; accepted 26 June 2008

\begin{abstract}
Corynebacterium pseudotuberculosis is the etiologic agent of caseous lymphadenitis, a disease that affects goats and sheep, and can cause severe economic losses. In this study, four different antigenic extracts were obtained from the attenuated strain T1, which was isolated in the state of Bahia (Brazil). Forty-four Canindé breed goats were divided in five groups, each receiving a different antigen solution and saline buffer as a control. The humoral response was monitored through the identification of specific IgG by indirect ELISA and Western Blotting, and the production of IFN- $\gamma$ was followed in order to observe the activation of cellular response. After twelve weeks of antigen inoculation, the animals were challenged with $2 \times 10^{5} \mathrm{CFU}$ of a wild strain, also isolated in Bahia, and necropsy was performed on all animals twelve weeks afterwards. It was observed that the attenuated bacteria gave a protection of $33.3 \%$, in addition to the weak humoral response elicited. Animals inoculated with secreted antigen associated with Freund's incomplete adjuvant and oligodeoxynucleotide containing unmethylated $\mathrm{CpG}$ dinucleotides (CpG ODN) showed a strong humoral response, but this inoculation could not prevent the spread of challenge bacteria in the majority of animals. These results demonstrate the immunogenic potential of the attenuated $\mathrm{T} 1$ strain in the development of a vaccine against caseous lymphadenitis in goats.
\end{abstract}

(C) 2008 Elsevier B.V. All rights reserved.

Keywords: Caseous lymphadenitis; Goats; C. pseudotuberculosis; CpG ODN; Vaccine

Abbreviations: TPP, three phase partitioning; FIA, Freund's incomplete adjuvant; BHI, brain and heart infusion; ODN, oligodeoxynucleotides.

* Corresponding author. Tel.: +55 713283 8899;

fax: +557132838899 .

E-mail addresses: lilia@ufba.br, lmouracosta@gmail.com (L.F. Moura-Costa).

\section{Introduction}

Caseous lymphadenitis is an infectious disease that affects small ruminants, particularly goats and sheep. The bacteria Corynebacterium pseudotuberculosis is the causative agent of the pathology (Batey, 1986; Brown and Olander, 1987; Williamson, 2001). The major clinical feature of this disease is the development of granulomas in peripheral and internal lymph nodes, containing necrotic tissue. In goats, other organs can be 
affected, such as lungs and liver. The disease exists worldwide (Sting et al., 1998; Pepin et al., 1999; Paule et al., 2003), and the bacteria can affect other species, like horses, bovines, but it rarely affects humans (Peel et al., 1997; Mills et al., 1997). C. pseudotuberculosis released from superficial abscesses persists in environment for a longtime and may become a source of contamination for the other animals in herd (Pepin et al., 1999). In the northeast region of Brazil, there is a high prevalence of the disease in goats, causing elevated economic losses, due to a reduction in weight gain and milk production, as well as problems in the commercialization of products derived from animal meat (Unanian et al., 1985; Brown and Olander, 1987; Brown et al., 1987).

Therapeutic treatment of the disease is not effective, as the pathogen has an intracellular location, and the distribution of drugs inside of the granuloma is poor. The puncture of the peripheral affected lymph nodes is the only viable treatment, but it can cause the spread of the bacteria in the environment, therefore elevating the risk of contamination (Nairn and Robertson, 1974). The internal granulomas are difficult to diagnose and may be a source of contamination for other animals (Ellis et al., 1987).

Several experimental trials have been developed in order to achieve a reliable vaccine to control the disease in sheep and goats. Different antigen preparations have been employed, such as formalin-killed bacterin, bacterial cellular wall and phospholipase D toxoid (Cameron et al., 1972; Brogden et al., 1984, 1996; Brown et al., 1986; Eggleton et al., 1991). An association of bacterin and formalin inactivated exotoxin was also tested, resulting in partial immunity characterized by fewer affected lymph nodes in each animal and fewer animals presenting with disease (Piontkowski and Shivvers, 1998).

In this study we used 44 Canindé breed goats from the state of Bahia in Brazil. Canindé is a naturalized Brazilian breed from northeastern Brazil and the experimental animals were from the same flock of purebreds. We tested the protective capacity and protection of crude $C$. pseudotuberculosis culture supernatant associated to FIA. In addition, we assessed the capacity of $\mathrm{CpG}$ ODN to improve the immune response and the protection against challenge of an antigen obtained through concentration of crude culture supernatant. Finally, we observed the capacity of a living attenuated strain to protect against challenge with a wild strain. The $C$. pseudotuberculosis $\mathrm{T} 1$ strain used in this experiment was isolated from a goat's lymph node, in the state of Bahia (Paule et al., 2004a). It is considered a natural, attenuated strain due to a weak synergistic hemolytic activity when co-cultivated with Rhodococcus equi and the absence of pathologies when susceptible BALB/c mice were inoculated with the strain (Vale et al., unpublished data), and is considered as an alternative in the development of a vaccine against caseous lymphadenitis.

\section{Methodology}

\subsection{Bacterial strains}

Two different strains of $C$. pseudotuberculosis were employed in this experiment. The strain T1, had its identification confirmed by Gram staining, colony morphology, synergistic hemolytic activity with CAMP factor of Rhodococcus equi, urease and catalase production. A commercial kit to perform a more reliable identification was also used (API Coryne BioMérieux). The pathogenic strain, employed as a challenge to vaccinated animals, was named VD57, and had a similar identification process as the attenuated strain. This pathogenic strain was isolated from a goat in the city of Juazeiro, Bahia State, Brazil.

\subsection{Antigens}

\subsubsection{Secreted antigen}

The C. pseudotuberculosis $\mathrm{T} 1$ strain was cultivated in Brain Heart Infusion (BHI) broth at $37^{\circ} \mathrm{C}$ for $72 \mathrm{~h}$. The culture was centrifuged for $30 \mathrm{~min}$ at $10,000 \times g$. Supernatant was filtered through a $0.22 \mu \mathrm{m}$ membrane filter. This supernatant was kept at $-20^{\circ} \mathrm{C}$ until use. The amount of protein was determined by Lowry's modified method (Bio-Rad). The protein concentration determined was $6.2 \mathrm{mg} / \mathrm{ml}$. This antigen was used in Group 1.

\subsubsection{Secreted antigen concentrated using three phase partitioning method (TPP)}

This antigen was obtained as previously described (Paule et al., 2004a), with some modifications. Briefly, the secreted antigen was prepared from culture supernatant saturated with $30 \%$ ammonium sulfate $\mathrm{pH} 4.0$ $(\mathrm{HCl})$ and $n$-butanol, under slow agitation at room temperature. The sample was homogenized, kept undisturbed for $60 \mathrm{~min}$, and centrifuged for $10 \mathrm{~min}$ at $1350 \times g$ at $4{ }^{\circ} \mathrm{C}$. The resulting interface was dissolved in small volumes of $20 \mathrm{mM}$ Tris buffer $\mathrm{pH} 7.4(500 \mu \mathrm{l}$ of buffer to $5 \mathrm{ml}$ of supernatant extract) followed by dialysis in $50 \mathrm{mM}$ phosphate buffer $\mathrm{pH} 7.4$ for $48 \mathrm{~h}$. Antigen was concentrated by ultra filtration with a 
$10 \mathrm{kDa}$ membrane (Millipore, USA) and filtered through a $0.22 \mu \mathrm{m}$ membrane filter. This supernatant was kept at $-20{ }^{\circ} \mathrm{C}$ until use. The amount of protein was determined by Lowry's modified method (Bio-Rad). This antigen was used in Groups 3 and 4.

\subsubsection{Somatic antigen}

The T1 C. pseudotuberculosis strain was cultivated in Brain Heart Infusion broth at $37{ }^{\circ} \mathrm{C}$ for $72 \mathrm{~h}$. The culture was centrifuged for $30 \mathrm{~min}$ at $3000 \times g$ and $4{ }^{\circ} \mathrm{C}$. The bacterial pellet was washed twice in PBS. It was homogenized in PBS (1:5) and sonicated at $60 \mathrm{~Hz}$, using five cycles of $60 \mathrm{~s}$ each (Branson Sonifier 450). The sample was centrifuged for $30 \mathrm{~min}$ at $10,000 \times \mathrm{g} /$ $4{ }^{\circ} \mathrm{C}$, and the supernatant was frozen at $-20{ }^{\circ} \mathrm{C}$ until use. This antigen was used in immunoblotting.

\subsubsection{Attenuated bacteria}

The T1 C. pseudotuberculosis strain was cultivated in BHI broth for $72 \mathrm{~h}$, and after this period, the bacterial concentration was adjusted to $2 \times 10^{6} \mathrm{CFU} / \mathrm{ml}$ with sterile PBS, and inoculated in animals from Group 2.

\subsection{5. $C p G O D N$}

A synthetic ODN containing unmethylated $\mathrm{CpG}$ dinucleotides (RW Genes, Brazil), with the following sequence TCGTCGTTGTCGTTTTGTCGTT (CpG motifs are underlined), was used in this study, which was previously used in sheep (Ioannou et al., 2002; Nichani et al., 2004).

\subsection{Animals and experimental groups}

Forty-four Canindé breed goats, age ranging from eight to ten months, were used as an experimental model. These animals were tested before the experiment for the presence of specific IgG against C. pseudotuberculosis (as described by Carminati et al., 2003) and caprine arthritis-encephalitis virus using a commercial kit (Biovetech). They were divided in five experimental groups, all of them receiving different subcutaneous doses of various antigens. Three groups received a vaccine booster with the same antigen preparation four weeks after the first dose. Goats were not segregated by groups, and they were maintained together for pasturing. Animals were fed with alfalfa hay, concentrated food and water ad libitum.

Group 1 (G1) ( $n=9$; three females and six males) immunized with $0.5 \mathrm{ml}$ of $C$. pseudotuberculosis strain T1 culture supernatant formulated with $0.5 \mathrm{ml}$ of Freund's incomplete adjuvant (Sigma-Aldrich). This supernatant was obtained from a Brain Heart Infusion broth culture, cultivated at $37^{\circ} \mathrm{C}$ for $72 \mathrm{~h}$. Animals received vaccine booster four weeks later.

Group 2 (G2) ( $n=9$; three females and six males) inoculated with $1 \mathrm{ml}$ of a suspension of $2 \times 10^{6} \mathrm{CFU} /$ $\mathrm{ml} \mathrm{T} 1$ strain bacteria in sterile phosphate buffer saline. This group did not receive a vaccine booster.

Group 3 (G3) ( $n=9$; two females and seven males) were immunized with a solution of $100 \mu \mathrm{g}$ of $C$. pseudotuberculosis $\mathrm{T} 1$ strain culture supernatant concentrated by TPP methodology in conjunction with $250 \mu \mathrm{g}$ of $\mathrm{CpG}$ TCGTCGTTGTCGTTTTGTCGTT (Nichani et al., 2004) and $0.5 \mathrm{ml}$ of FIA and sterile PBS in a total volume of $1 \mathrm{ml}$. Four weeks later, goats were re-immunized with the same formulation.

Group 4 (G4) $(n=10$; three females and seven males) were inoculated with the $1 \mathrm{ml}$ of the same solution employed in Group 3 without FIA. A fourweek interval was allowed between the first and second immunizations.

Group 5 (G5) ( $n=7$; one female and six males) represented the control of the experiment, and were only inoculated with $1 \mathrm{ml}$ of sterile PBS and did not receive a second inoculation.

Blood samples were collected at each week postimmunization day.

\subsection{Experimental challenge}

All animals from experimental groups were challenged with C. pseudotuberculosis wild strain (VD57) after twelve weeks from the first dose of the vaccinal inoculum. The infection was carried out through a double inoculation of $1 \times 10^{5} \mathrm{CFU}$ C. pseudotuberculosis in the inguinal region (intradermally on the right side, and trickle on scarified skin on the left side).

\subsection{Indirect ELISA for detection of specific IgG against $C$. pseudotuberculosis}

The indirect ELISA assay for the identification of $C$. pseudotuberculosis specific immunoglobulins in goats was performed as described before (Carminati et al., 2003; Paule et al., 2003). Briefly, EIA plates (Costar) were sensitized with $100 \mu \mathrm{l}$ per well of C. pseudotuberculosis strain $\mathrm{T} 1$ supernatant diluted 1:100 in $0.05 \mathrm{M}$ carbonate-bicarbonate buffer, $\mathrm{pH} 9.6$, and incubated at $4{ }^{\circ} \mathrm{C}$ overnight. After two washes with PBS Tween $0.05 \%$ (PBST), plates were blocked with $200 \mu$ per well of 5\% dry skimmed powder milk in PBST for $2 \mathrm{~h}$ at $37^{\circ} \mathrm{C}$. After this, $100 \mu \mathrm{l} /$ well of sera samples diluted $1: 100$ in $1 \%$ dry skimmed powder milk PBS-T were added and incubated $1 \mathrm{~h}$ at $37^{\circ} \mathrm{C}$. Plates were then 
washed with PBS-T, and incubated with rabbit anti-goat IgG peroxydase conjugated (Sigma Cat. No. A-5420) 1:40,000 in PBS-T $1 \%$ dry skimmed powder milk for $45 \mathrm{~min}$ at $37^{\circ} \mathrm{C}$. After five additional washes, plates were developed with orthopenylenediamine (OPD) and results were read at $490 \mathrm{~nm}$.

\subsection{Interferon-gamma quantification}

The quantification of IFN- $\gamma$ in blood cultures was performed in samples collected from the experimental animal groups as previously described (Paule et al., 2003; Meyer et al., 2005), employing Bovigam Kit (CSL, Australia), with some modifications. Briefly, $2 \mathrm{~h}$ after collection, heparinized blood was incubated at $37{ }^{\circ} \mathrm{C}$ for $48 \mathrm{~h}$ in a humidified atmosphere of $5 \% \mathrm{CO}_{2}$ in a 24 well culture tray with $20 \mu \mathrm{g}$ of secreted antigen in chemically defined medium (Moura-Costa et al., 2002), $5 \mu l$ of pokeweed mitogen as positive control, or PBS as negative control. After this period, blood was centrifuged and IFN- $\gamma$ was quantified in plasma employing a sandwich ELISA (Bovigam). OD results were converted to IFN- $\gamma$ indices as describe by Paule et al. (2003):

IFN- $\gamma$ indices

$$
=\frac{\text { OD sample }- \text { OD negative control }}{\text { OD positive control }- \text { OD negative control }} \text {. }
$$

\subsection{Immunoblotting}

An immunoblotting methodology was developed in order to determine $C$. pseudotuberculosis antigens recognized by specific immunoglobulins produced by the animals in days 14, 21 and 84 after immunization, and 14, 21 and 84 after challenge. A sonicated antigen from $\mathrm{T} 1$ strain cultivated in $\mathrm{BHI}$ broth, at $37{ }^{\circ} \mathrm{C}$ for $48 \mathrm{~h}$, was separated by a discontinuous SDS-PAGE system with a $4 \%$ stacking gel and a 10\% running gel. Proteins were transferred to a nitrocellulose membrane. This membrane was blocked overnight at $4{ }^{\circ} \mathrm{C}$ with dry skimmed milk 5\% diluted in $0.05 \%$ PBS-T. Afterwards, an incubation with serum samples diluted 1:50 in PBS$\mathrm{T}$ containing $1 \%$ of dry skimmed powder milk was performed at $37^{\circ} \mathrm{C}$ for $1 \mathrm{~h}$. After five washes with PBS$\mathrm{T} 0.05 \%$, a rabbit anti-goat IgG horseradish peroxydase conjugated (Sigma Cat. No. A-5420) diluted 1:100 in PBS-T was added and incubated for $1 \mathrm{~h}$ at $37{ }^{\circ} \mathrm{C}$. After five additional washes, the membrane was developed with a solution of $0.3 \%$ 4-chloro-1-naphtol diluted 1:5 in PBS and $10 \mu \mathrm{l}$ of hydrogen peroxide. The reaction was then interrupted and membranes scanned.

\subsection{Post-mortem examination}

Twenty-four weeks after the beginning of the experiment, all animals were sacrificed and analyzed for occurrence of typical caseous lymphadenitis lesions. Every abdominal and thoracic organ was screened, but special attention was given to lungs, liver, spleen and lymph nodes. The tissues that presented lesions compatible with $C$. pseudotuberculosis infection were collected in sterile vials and flasks with $10 \%$ formalin. The tissues were embedded in paraffin, allowing the confection of histology slides, and stained with hematoxylin-eosin method.

\subsection{Bacterial identification}

Tissue samples aseptically collected in necropsy were disrupted in PBS and plated onto BHI blood Agar, and the bacterial identification was made by analysis of colony morphology, Gram stain, catalase and urease activity, carbohydrate fermentation (glucose, lactose, sucrose and mannose) and synergistic hemolytic activity with CAMP factor of Rhodococcus equi.

\subsection{Statistical analysis}

The results of the ELISA for quantification of IFN- $\gamma$ and determination of specific IgG against C. pseudotuberculosis were screened by variance analysis, and when statistical significance was found, the mean correlation was made by Tukey test, for significant statistical differences when $p<0.05$. The results from the post-mortem examination were treated by Pearson's Chi-square test.

\section{Results}

\subsection{Indirect ELISA for C. pseudotuberculosis specific IgG determination}

Means of optical densities (O.D.) by group are represented in Fig. 1. It was possible to detect a significant difference $(p<0.05)$ between the means of Groups 1 and 3 relative to the other groups. Group 4 presented an intermediary result, as Groups 2 and 5 showed the lowest levels of IgG, without a statistical difference between them.

Means of optical density after the challenge with the wild strain are shown in Fig. 2. Groups 1 and 3 presented the highest means, without statistical difference between them, however Group 5 presented, as expected, the lowest OD, when compared to another groups. 


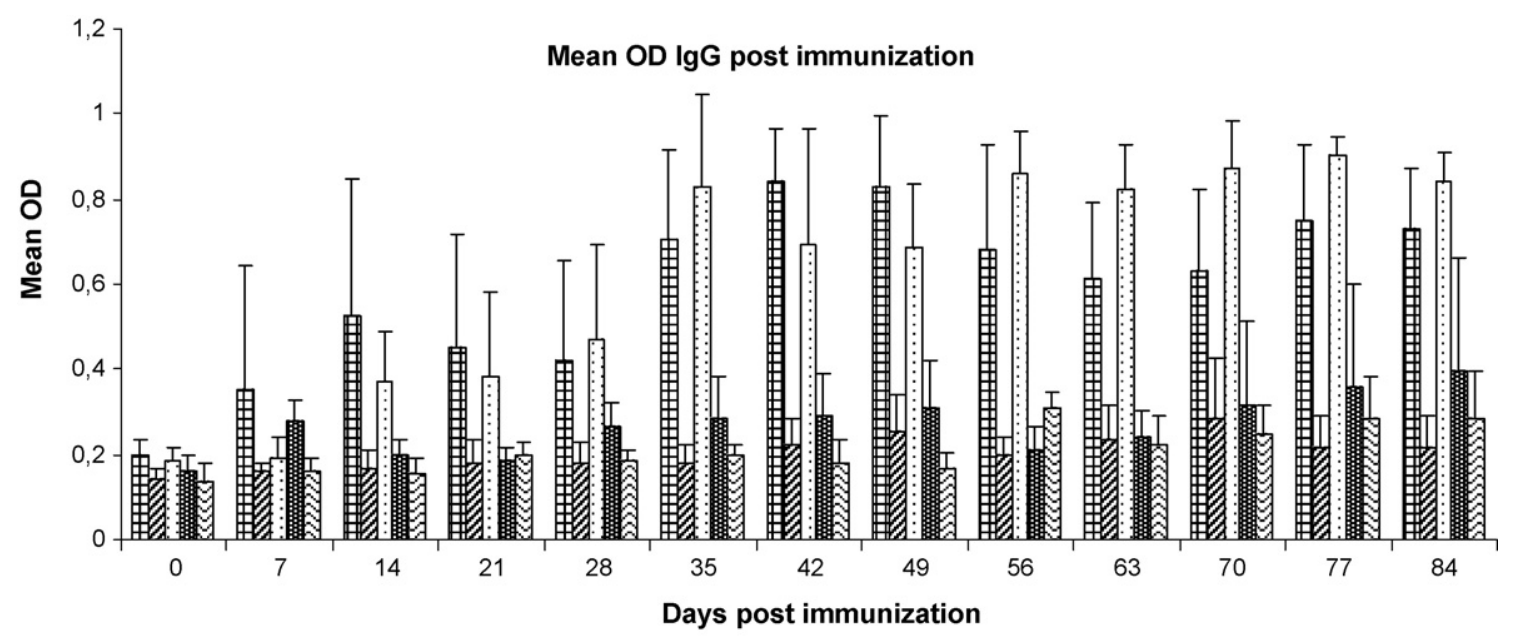

田GROUP 1 GROUP 2 GGROUP 3 睡GROUP 4 娄GROUP 5

Fig. 1. Mean and standard error $\left(\mathrm{OD}_{490 \mathrm{~nm}}\right)$ of humoral response to C. pseudotuberculosis excreted-secreted antigens of groups after immunization. Group 1 supernatant in BHI broth + FIA; Group 2 live T1 strain; Group 3 supernatant concentrated by TPP method + FIA + CpG; Group 4 supernatant concentrated by TPP method + CpG without FIA; Group 5 PBS (control). Groups 1, 3 and 4 were re-immunized 28 days after first immunization.

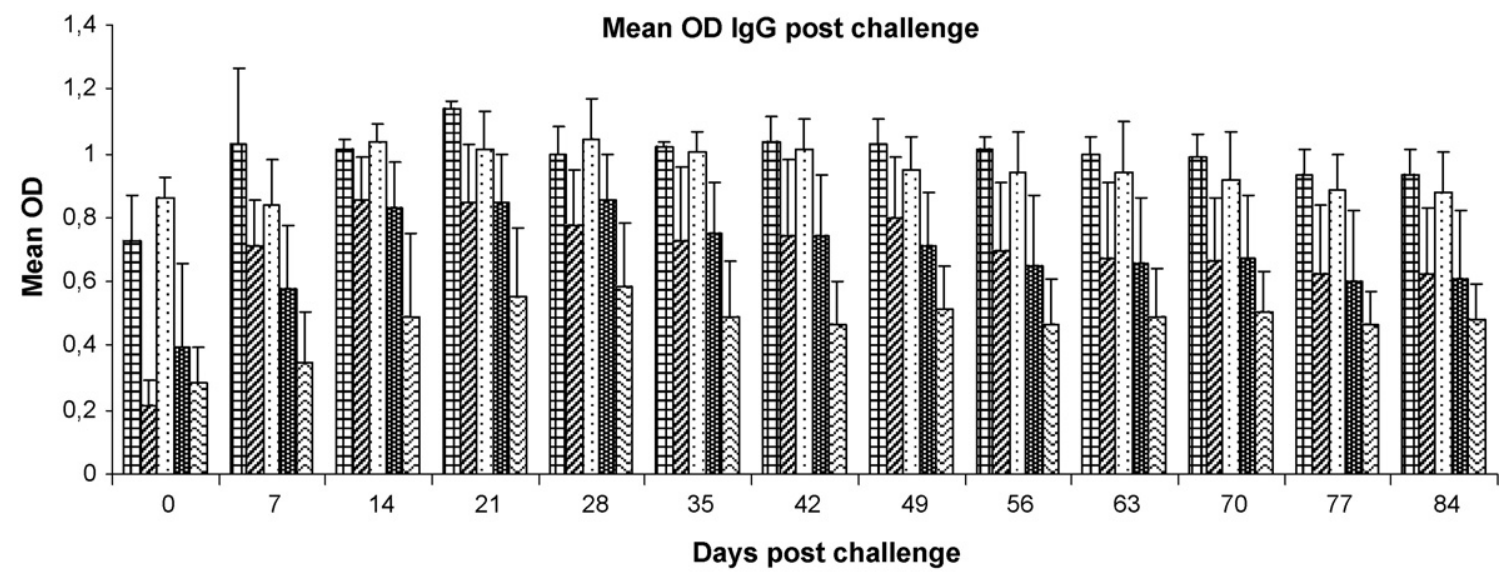

日GROUP 1 日GROUP 2 GGROUP 3 目GROUP 4 目GROUP 5

Fig. 2. Mean and standard error of $\mathrm{OD}_{490 \mathrm{~nm}}$ (IgG ELISA) per group after challenge (day 0 ) with a wild strain $\left(2 \times 10^{5} \mathrm{CFU}\right)$. Group 1 supernatant in BHI broth + FIA; Group 2 live T1 strain; Group 3 supernatant concentrated by TPP method + FIA + CpG; Group 4 supernatant concentrated by TPP method $+\mathrm{CpG}$ without FIA; Group 5 PBS (control).

Tables 1 and 2 show the OD means in four defined moments of the study. It was possible to observe that the humoral response in Groups 1 and 3 was higher than other groups, in the post-immunization period, during the post-challenge time, these groups still had intense production of IgG. Groups 2 and 4 had weak OD results, but higher than that found in the control group (G5). ANOVA and Tukey statistical tests were employed to analyze the ELISA results.

\subsection{Interferon-gamma responses}

The OD means comparison in the post-immunization quantification of IFN- $\gamma$ is presented in Fig. 3. Groups 1 and 3 presented the highest values, however without significant statistical difference between them. In the post-challenge period (Fig. 4), theses groups still had the highest production of the cytokine, but also Group 5 had high values, not statistically different from Groups 
Table 1

Optical densities (OD) means found in C. pseudotuberculosis specific $\operatorname{IgG}$ ELISA at 21th and 42th days post immunization (PI)

\begin{tabular}{lll}
\hline GROUP & OD means at 21th PI & OD means at 42th PI \\
\hline G1 & $0.4537^{\mathrm{a}}$ & $0.8382^{\mathrm{c}}$ \\
G2 & $0.1819^{\mathrm{b}}$ & $0.2198^{\mathrm{d}}$ \\
G3 & $0.3858^{\mathrm{a}, \mathrm{b}}$ & $0.6909^{\mathrm{c}}$ \\
G4 & $0.1855^{\mathrm{b}}$ & $0.2926^{\mathrm{d}}$ \\
G5 & $0.1985^{\mathrm{b}}$ & $0.2057^{\mathrm{d}}$ \\
\hline
\end{tabular}

Statistical analysis was made using ANOVA and Tukey tests $(p<0.05)$. Different superscript letters stands for significant statistical differences among groups.

${ }^{\text {a }}$ Group 1 showed statistically significant difference compared to all other groups $(p<0.05)$ except group 3 .

b There is no statistically significant difference between Group 3 and the other groups $(p<0.05){ }^{\mathrm{c}, \mathrm{d}}$ Groups 1 and 3 showed statistically significant difference compared to the other groups, but there is no difference between each other $(p<0.05)$.

Table 2

Optical densities (OD) means found in C. pseudotuberculosis specific IgG ELISA at 21th and 42th days post challenge (PC)

\begin{tabular}{lll}
\hline GROUP & OD means at 21th PC & OD means at 42th PC \\
\hline G1 & $1.1411^{\mathrm{a}}$ & $1.0398^{\mathrm{d}}$ \\
G2 & $0.8478^{\mathrm{b}}$ & $0.7468^{\mathrm{e}}$ \\
G3 & $1.0121^{\mathrm{a}, \mathrm{b}}$ & $1.0115^{\mathrm{d}}$ \\
G4 & $0.8479^{\mathrm{b}}$ & $0.7404^{\mathrm{e}}$ \\
G5 & $0.5506^{\mathrm{c}}$ & $0.4684^{\mathrm{f}}$
\end{tabular}

Statistical analysis was made using ANOVA and Tukey tests $(p<0.05)$. Different superscript letters stands for significant statistical differences among groups.

${ }^{\text {a }}$ Group 1 showed statistically significant difference compared to the other groups $(p<0.05)$.

b There is no statistically significant difference between Group 3 and Groups 2 and $4(p<0.05) \cdot{ }^{\mathrm{d}, \mathrm{e}, \mathrm{f}}$ Groups 1 and 3 showed statistically significant difference compared to the other groups, but there is no difference between each other $(p<0.05)$.

1 and 3. On the other hand, there were no significant differences between Groups 5 and 2, when compared to Group 4 results.

\subsection{Immunoblotting}

The immunoblotting analyses, as shown in Tables 3 and 4, demonstrated that there was a less intense recognition of $C$. pseudotuberculosis antigens immediately after the immunization, and the number of recognized protein bands grew in the five groups with time. After the challenge, it could be demonstrated that twenty-four antigens had been well-recognized; ranging from 12 to $125 \mathrm{kDa}$. The most frequently recognized antigens on the fourteenth day after immunization had the molecular weight of $31 \mathrm{kDa}$ (in all groups except control) and $67 \mathrm{kDa}$ that was recognized by Groups 1, 2, 4 and the control group. In the twenty-first day after immunization, there was an increase in the detection of the $31 \mathrm{kDa}$ band in the immunized groups, which also occurred in the proteins with 58, 67, 85 and $100 \mathrm{kDa}$. In the fourteenth day after challenge, all groups presented recognition of nine antigens: $12,16,21,31,40,58,78$, 92 e $108 \mathrm{kDa}$. The $48 \mathrm{kDa}$ antigen was only recognized by the immunized groups. The $31 \mathrm{kDa}$ antigen was recognized by $100 \%$ of Groups 1, 2 and 3 animals, $90 \%$ from Groups 4 and $57.1 \%$ from the control group. The number of recognized antigens and their intensity increased until the eighty-fourth day after challenge.

\subsection{Post-mortem examination}

Four patterns of lesions were considered in the necropsy examination: the first (P1) stands for the complete absence of infected tissues, the second (P2) represents animals with lesions only in the site of challenge, the third (P3) represents infection in the site of challenge and in another tissue, and the last pattern (P4) is characterized by the absence of infection in the site of challenge, but presenting lesions in other tissues. These results are shown in Table 5. Twenty-five percent of G1 group did not present lesions (P1) and 75\% showed pathologic lesions in other tissues (P4). In Group 2, $33.3 \%$ of all animals did not present any lesions (P1), and there was $22.2 \%$ in each of the other lesion patterns. $22.2 \%$ of experimental animals from Group 3 did not have any lesions (P1), $11.1 \%$ showed lesions only in the challenge site (P2), $11.1 \%$ showed lesions in the bacteria inoculation region and in other tissue (P3) and $55.6 \%$ had lesions in organs distant from the inoculation area (P4). In the Group 4 animals, $70 \%$ presented the pattern 3 of lesions, and 30\% the pattern 4. Finally, the Group 5, our control group, surprisingly had $14.3 \%$ with no pathological findings, but $28.6 \%$ presented the lesion pattern 3 and $57.1 \%$ with pattern 4 . Chi-square test showed a significant statistical difference $(p=0.047)$ among the immunization schedules and the lesion patterns. All lesions found in necropsy had positive results for the presence of C. pseudotuberculosis in bacterial identification, by previously described biochemical assays. There was strong evidence that the mean percentage (by variance analysis) of tissues infected was smaller in Group 2 than in the other groups.

\section{Discussion}

This study was conducted with the aim to evaluate the humoral and cellular response in Canindé goats 


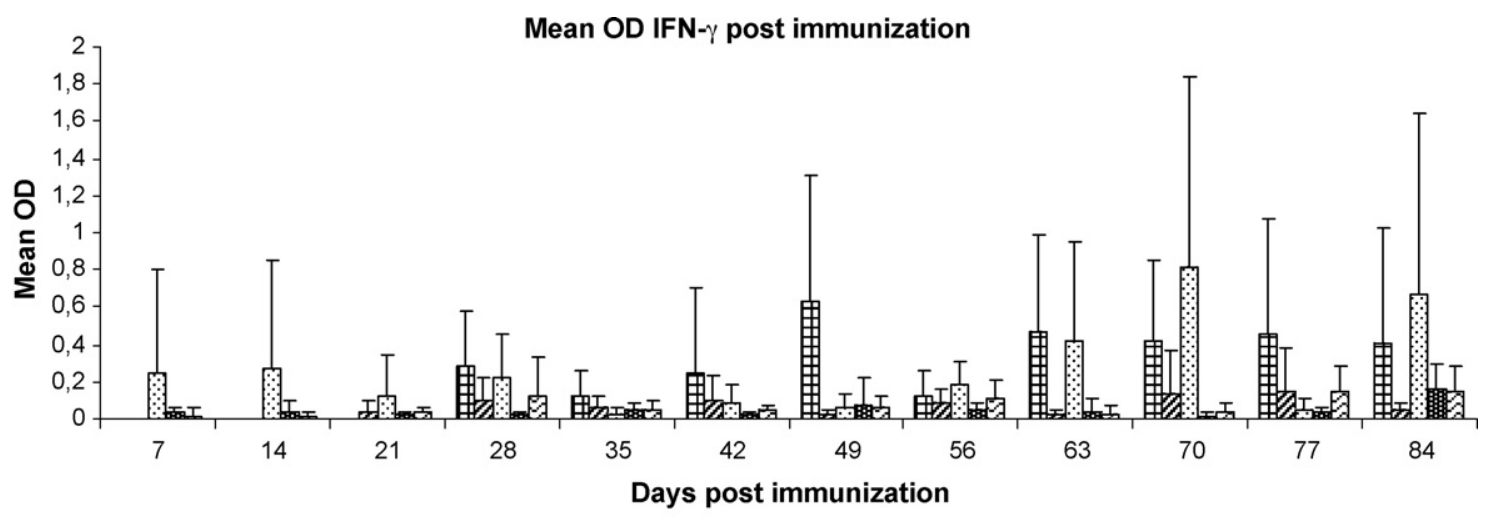

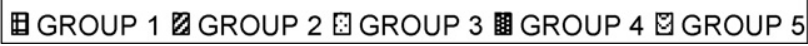

Fig. 3. Mean and standard error of OD IFN- $\gamma$ ELISA, per group, in post immunization period. Group 1 supernatant in BHI broth + FIA; Group 2 live T1 strain; Group 3 supernatant concentrated by TPP method + FIA + CpG; Group 4 supernatant concentrated by TPP method + CpG without FIA; Group 5 PBS (control). Groups 1, 3 and 4 were re-immunized 28 days after first immunization. Groups 3, 4 and 5 had blood collected from day 7 post immunization. Group 2 had blood collected from day 21 and Group 1 from day 28 after immunization.

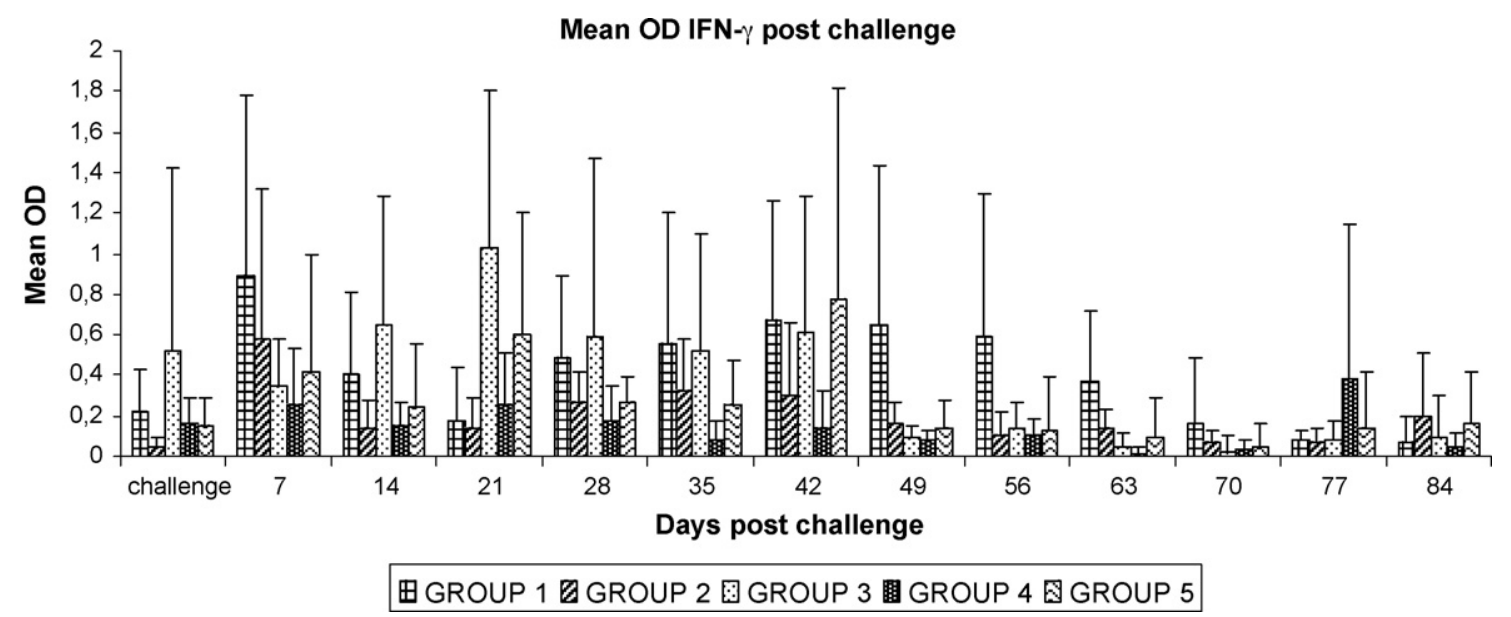

Fig. 4. Mean and standard error of OD IFN- $\gamma$ ELISA, per group, in post challenge period. Challenge at day 0 with $C$. pseudotuberculosis wild strain $\left(2 \times 10^{5} \mathrm{CFU}\right)$. Group 1 supernatant in BHI broth + FIA; Group 2 live T1 strain; Group 3 supernatant concentrated by TPP method + FIA + CpG; Group 4 supernatant concentrated by TPP method + CpG without FIA; Group 5 PBS (control).

against antigens from an attenuated strain of $C$. pseudotuberculosis. This strain was isolated in the western region of the state of Bahia, (Brazil), and has been the subject of studies in our laboratory for seven years. Since there were no pathogenic lesions in BALB/ c mice inoculated with $\mathrm{T} 1$ strain $\left(10^{7} \mathrm{CFU}\right)$ or its culture supernatant in BHI broth, we decided to study their immunoprotective effect. We employed both living bacteria as well as its culture supernatant concentrate, the last antigen being associated with CpG ODN of known in-vitro and in-vivo biological activity (Rankin et al., 2001; Nichani et al., 2004) as an adjuvant. Goats were the subject of our study, since there have already been many studies relating to caseous lymphadentis in sheep (Cameron et al., 1972; Eggleton et al., 1991; Piontkowski and Shivvers, 1998; Hodgson et al., 1999; Fontaine et al., 2006), but not many studies of the caprine model (Brown et al., 1986).

The indirect ELISA results showed that the humoral response was higher in the animals immunized with culture supernatant (G1) and in the animals inoculated with a concentrated supernatant culture obtained by using a three-phase partitioning technique (Paule et al., 2004a) associated with CpG (ODN) and FIA (G3). The present study confirmed the results of Ioannou et al. (2002), who related that the use of $\mathrm{CpG}$ alone elicits an immune response less intense than in conjunction with other adjuvants (G3 and G4). It was significant that 
Table 3

Antigenic recognition by treatment groups post immunization (PI) (in \%)

\begin{tabular}{|c|c|c|c|c|c|c|c|c|c|c|c|c|c|c|c|}
\hline \multirow[t]{2}{*}{ Antigen (kDa) } & \multicolumn{3}{|c|}{ Group 1} & \multicolumn{3}{|c|}{ Group 2} & \multicolumn{3}{|c|}{ Group 3} & \multicolumn{3}{|c|}{ Group 4} & \multicolumn{3}{|c|}{ Group 5} \\
\hline & 14 & 21 & 84 & 14 & 21 & 84 & 14 & 21 & 84 & 14 & 21 & 84 & 14 & 21 & 84 \\
\hline 125 & & & & & & & & 33.3 & 33.3 & 40 & 20 & 10 & & & 28.6 \\
\hline \multicolumn{16}{|l|}{120} \\
\hline \multicolumn{16}{|l|}{116} \\
\hline 112 & & & & & & & & & & & & & & & 28.6 \\
\hline 108 & & & & & & & & & & & & 20 & & & \\
\hline 100 & & 22.2 & 66.7 & & & & & 44.4 & 22.2 & & 30 & 20 & & & 28.6 \\
\hline 92 & & 44.4 & & 44.4 & & & & & 22.2 & & & & & & \\
\hline 85 & & & & & 22.2 & & & 44.4 & & & 40 & & & & \\
\hline 78 & & & 44.4 & & & 33.3 & & 33.3 & 22.2 & & & 20 & & 28.6 & 28.6 \\
\hline 73 & & & & & & & & & & & 20 & & & & \\
\hline 67 & & & & 55.6 & & 33.3 & & 33.3 & 100 & & 30 & & & & 28.6 \\
\hline \multicolumn{16}{|l|}{64} \\
\hline 58 & 22.2 & & & 77.8 & & & & 22.2 & & & & & & & \\
\hline \multicolumn{16}{|l|}{53} \\
\hline 48 & & & 22.2 & & & & & & 44.4 & & & 20 & & & \\
\hline 43 & & & & & 55.6 & 22.2 & & 22.2 & & & & & & & \\
\hline 40 & & & 77.8 & & & & & 22.2 & 77.8 & & & & & & \\
\hline 36 & & & & & & & & & & & & & & & 28.6 \\
\hline 31 & 100 & 88.9 & 88.9 & 100 & 100 & 44.4 & & 100 & 100 & 30 & 70 & 40 & & & \\
\hline 28 & & & 77.8 & & & & & & 88.9 & & & & & & \\
\hline \multicolumn{16}{|l|}{24} \\
\hline 21 & & & & 66.7 & & 22.2 & & & & & & & & & \\
\hline 16 & & & 22.2 & & & & 33.3 & 22.2 & 55.6 & & & & & & \\
\hline 12 & & 22.2 & & & & & & & 66.7 & & & & & & \\
\hline
\end{tabular}

Table 4

Antigenic recognition by treatment groups post challeng (PC) (in \%)

\begin{tabular}{|c|c|c|c|c|c|c|c|c|c|c|c|c|c|c|c|}
\hline \multirow[t]{2}{*}{ Antigen (kDa) } & \multicolumn{3}{|c|}{ Group 1} & \multicolumn{3}{|c|}{ Group 2} & \multicolumn{3}{|c|}{ Group 3} & \multicolumn{3}{|c|}{ Group 4} & \multicolumn{3}{|c|}{ Group 5} \\
\hline & 14 & 21 & 84 & 14 & 21 & 84 & 14 & 21 & 84 & 14 & 21 & 84 & 14 & 21 & 84 \\
\hline 125 & 33.3 & 44.4 & & & 100 & & & & & & & & & 100 & \\
\hline 120 & & 100 & & & & & & & & & & & & & \\
\hline 116 & & & & & & & & & & & & & & 85.7 & \\
\hline 112 & 22.2 & 88.9 & 100 & & 77.8 & 88.9 & & & 77.8 & & & & & & \\
\hline 108 & 77.8 & & & 55.6 & & 22.2 & & & & 40 & 100 & 100 & 42.9 & & \\
\hline 100 & 55.6 & 77.8 & 100 & & 100 & 77.8 & & 100 & 77.8 & 50 & & & 28.6 & 85.7 & 10 \\
\hline 92 & & 77.8 & 88.9 & 33.3 & 44.4 & 44.4 & 44.4 & 77.8 & 88.9 & 40 & 100 & 90 & 42.9 & & 80 \\
\hline 85 & 55.6 & & 77.8 & & & 88.9 & 33.3 & & 22.2 & & & 40 & & & \\
\hline 78 & 66.7 & 55.6 & 88.9 & 77.8 & 88.9 & 88.9 & 66.7 & 77.8 & 77.8 & 50 & 70 & 100 & 71.4 & 100 & 100 \\
\hline 73 & 66.7 & 77.8 & 66.7 & & 22.2 & & & 66.7 & 55.6 & 50 & 90 & 30 & 42.9 & & 100 \\
\hline 67 & & 33.3 & 55.6 & & 44.4 & 77.8 & 55.6 & 66.7 & 44.4 & 40 & 20 & 40 & 57.1 & & 60 \\
\hline 64 & 22.2 & 88.9 & 100 & & 77.8 & 100 & 44.4 & & 66.7 & 30 & 40 & 50 & 28.6 & & \\
\hline 58 & 22.2 & 66.7 & 77.8 & 33.3 & 44.4 & & 44.4 & 77.8 & 77.8 & 40 & 40 & 100 & 57.1 & 71.4 & 60 \\
\hline 53 & & & 44.4 & & & 66.7 & & 44.4 & & & 70 & & & & \\
\hline 48 & 33.3 & 66.7 & 44.4 & 77.8 & 66.7 & 100 & 88.9 & 88.9 & 88.9 & 30 & 90 & 50 & & & 100 \\
\hline 43 & 33.3 & 44.4 & 33.3 & & 44.4 & 22.2 & & & & 80 & & 20 & & & \\
\hline 40 & 55.6 & 22.2 & 55.6 & 88.9 & 44.4 & 44.4 & 55.6 & & 66.7 & 20 & & 80 & 57.1 & 100 & 60 \\
\hline 36 & & & 66.7 & & & & & 33.3 & 77.8 & & 20 & & & & \\
\hline 31 & 100 & 100 & 77.8 & 100 & 77.8 & 88.9 & 100 & 100 & 66.7 & 90 & 100 & 90 & 57.1 & 28.5 & 80 \\
\hline 28 & & 44.4 & 33.3 & & 55.6 & 77.8 & 33.3 & 33.3 & & 20 & 20 & 50 & 57.1 & & 20 \\
\hline 24 & & & 88.9 & & & 77.8 & & & 44.4 & & 70 & & & & \\
\hline 21 & 33.3 & 77.8 & 66.7 & & 77.8 & 100 & 33.3 & 88.9 & 100 & 80 & 50 & 100 & 100 & 100 & 100 \\
\hline 16 & 33.3 & 44.4 & 100 & 66.7 & 33.3 & 77.8 & 100 & 88.9 & 100 & 70 & 100 & 40 & 85.7 & 57.1 & 80 \\
\hline 12 & 33.3 & 88.9 & 77.8 & 100 & 100 & 66.7 & 100 & 77.8 & 44.4 & 100 & 90 & 80 & 100 & 85.7 & 20 \\
\hline
\end{tabular}


Table 5

Summary of post mortem examination results showing groups and percentage of pattern of infection

\begin{tabular}{lccccc}
\hline & Group 1 & Group 2 & Group 3 & Group 4 & - \\
\hline P 1 & $25 \%$ & $33.3 \%$ & $22.2 \%$ & - & - \\
P 2 & - & $22.2 \%$ & $11.1 \%$ & $7.3 \%$ \\
P 3 & - & $22.2 \%$ & $11.1 \%$ & $70 \%$ & $28.6 \%$ \\
P 4 & $75 \%$ & $22.2 \%$ & $55.6 \%$ & $30 \%$ & $57.1 \%$ \\
Total & 100 & 100 & 100 & 100 & 100 \\
\hline
\end{tabular}

P 1: No tissues infected. P 2: Infection in the inoculation site but no other tissues. P 3: Infection in the inoculation site and at least one other tissue. P 4: Infection in other tissue but not in the inoculation site. Obs: in the moment of the necropsy, due to problems with animal handling, it was not possible to perform the post-mortem analysis of one animal from Group 1, so $n$ for necropsy in Group 1 is 8 animals.

animals inoculated with the attenuated strain $\mathrm{T} 1$ did not develop a significant humoral response, maintaining an OD mean next to the control group (G5) and under the cut-off established for the assay (0.250). However, Group 2 animals showed an elevation in the levels of specific immunoglobulins in the post-challenge period. This situation indicates that $\mathrm{T} 1$ strain was capable of inducing an immunologic response, even without any initial significant antibody production.

Immunoblotting results showed that animals that did not receive any antigenic stimulation before challenge (G5) were not able to recognize a $31 \mathrm{kDa}$ antigen, which is compatible with bacterial exotoxin phospholipase D (Songer et al., 1990; Hodgson et al., 1990). It can also be seen that, before challenge, analyzing the three study points $(14,21$ and $84 \mathrm{PI}), 100 \%$ of animals from Groups 1,2 and 3 were capable of producing antibodies that recognized this $31 \mathrm{kDa}$ band in, at least, one of these points. Interestingly, there is a $67 \mathrm{kDa}$ antigen that is recognized by all experimental animals (Table 3 ). This immunorecognition has been described by other authors (Sting et al., 1998; Paule et al., 2003), and may correspond to a chaperonin (Ellis et al., 1991). Another antigen, a $40 \mathrm{kDa}$ band, was described as a serineprotease (Walker et al., 1994; Wilson et al., 1995) and is predominately recognized in animals immunized with secreted antigens from supernatant culture (G1 and G3), but not in G5. The antigen used in Western-Blot was a sonicated whole bacteria, called somatic antigen, and the presence of specific immunoglobulins against these protein bands has been documented in other author's research on infected animals (Ellis et al., 1991; Sting et al., 1998; Paule et al., 2004b).

Using a commercial kit to identify bovine IFN- $\gamma$, it was observed that the levels of this cytokine in blood cultures stimulated with secreted-excreted antigens were low after immunization. This situation was also described by Prescott et al. (2002) and demonstrated that bacterin/toxoid-vaccinated sheep do not express high levels of INF- $\gamma$ when stimulated by somatic antigen. Interestingly, groups that presented an elevated humoral response (G1 and G3) also had higher IFN- $\gamma$ levels. Furthermore, in the post-challenge period, Group 1 presented the highest IFN- $\gamma$ levels again. Although this was a detectable humoral and cellular response, these groups did not present the best immunoprotection results after challenge. All groups presented, as expected, a cellular immune response higher in the post-challenge period. We observed that there is a fluctuation in the individual cellular response, and these findings are in agreement with previous studies and may be partially explained by genetic differences or bacterial shedding (Prescott et al., 2002; Paule et al., 2003; Meyer et al., 2005).

The statistical analysis of post-mortem examination showed a significant difference among experimental groups. $33.3 \%$ of Canindé goats immunized with T1 strain (G2) did not present any pathological alteration (P1). When considering lesions only in the challenge site (P2) as a partial immunoprotection, we can consider that Group 2 showed $55.55 \%$ of immunoprotection against challenge $(\% \mathrm{P} 1+\% \mathrm{P} 2)$. When associating humoral response results with post-mortem findings, it can be seen that high antibody levels were not sufficient to induce protection, either in the site of infection, or in distant tissues. Although sera of animals in immunized groups were able to recognize the $31 \mathrm{kDa}$ band related to phospholipase $\mathrm{D}$, these immunoglobulins were not sufficient to avoid the spread of $C$. pseudotuberculosis from the inoculation site. Because the secreted antigens contained other proteins, the humoral responses against them did not protect animals against challenge. This is in agreement with previous studies conducted in goats vaccinated with toxoid associated with FIA that offered a partial protection, with a reduction in the number of granulomas after challenge, but not a complete protection (Brown et al., 1986). It needs to be highlighted that the pre-challenge 
titre of antibody against phospholipase D is not sufficient to confer protection (Hodgson et al., 1999). We could not find any pathological lesion in one animal from the control group (G5). This situation also occurs in other vaccine trials (Eggleton et al., 1991; Hodgson et al., 1999) where some animals challenged with $C$. pseudotuberculosis did not developed typical lesions of the disease.

Interestingly, the group inoculated with the attenuated strain (G2) showed the best results in immunoprotection, among all groups. There were no local or systemic reactions to the $2 \times 10^{6} \mathrm{CFU}$ inoculum, and no booster was administered. In addition to the initial weak antibody production, there was a significant humoral response after challenge, suggesting that the inoculated dose had primed the animals. Simmons et al. (1998), observed that sheep vaccinated with attenuated mutants of $C$. pseudotuberculosis were not able to clear the bacteria from the lymph node draining the inoculation site. Considering that attenuated $\mathrm{T} 1$ strain can persist for a long time in the inoculation in site, it is possible that the immunogenic stimulus may have persisted, activating the cellular and humoral response more efficiently. Many studies have demonstrated the importance of the cellular immune response against $C$. pseudotuberculosis (Pepin et al., 1997; Hodgson et al., 1999). Although our experiments did not demonstrate an expressive cellular response in the animals inoculated with $\mathrm{T} 1$ strain after immunization, efforts have been undertaken in order to establish a better stimulating antigen and a Real Time PCR, in order to follow cytokine kinetic production. In addition to its immunoprotection, the $\mathrm{T} 1$ attenuated strain can be seen as a promising option, due to the low cost and protective characteristics of a single dose.

\section{Conclusions}

The use of a culture supernatant associated with FIA, and TPP concentrated secreted proteins combined with $\mathrm{CpG}$ and FIA, stimulated a strong humoral response, but without robust disease prevention. Immunization with TPP antigenic solution associated with $\mathrm{CpG}$, but without FIA, did not cause a reliable protection and was statistically equal to the control group. From the four antigenic preparations, the attenuated strain showed the best results, however with a low humoral response. It will be necessary to adjust the challenge dose and utilize a more efficient method of studying the cellular response in future research to have a better understanding of this potential vaccine strain

\section{Acknowledgements}

The authors are sincerely grateful to the technical staff of the LABIMUNO/ICS for their assistance. We also thank Sr. Joãozito Andrade for the supply of Canindé goats and Dr. Arthur Hage for supply of T1 strain used in this experiment. This study was supported by Laboratory of Immunology of Health Sciences Institute of Federal University of Bahia (Laboratório de Imunologia do Instituto de Ciências da Saúde da Universidade Federal da Bahia - LABIMUNO/ICS/UFBA) and by the Scientific and Technological Development Fund (Fundo de Desenvolvimento Científico e Tecnológico - FUNDECI) of Banco do Nordeste do Brasil S. A. (BNB).

\section{References}

Batey, R.G., 1986. Pathogenesis of caseous lymphadenitis in sheep and goats. Aust. Vet. J. 63, 269-272.

Brogden, K.A., Cutlip, R.C., Lehmkuhl, H.D., 1984. Comparison of protection induced in lambs by Corynebacterium pseudotuberculosis whole cell and cell wall vaccines. Am. J. Vet. Res. 45, 2393-2395.

Brogden, K.A., Glenn, J.S., East, N., Audibert, F., 1996. A Corynebacterium pseudotuberculosis bacterin with muramyl dipeptide induces antibody titers, increases the time of onset, and decreases naturally occurring external abscesses in sheep and goats. Small Rumin. Res. 19, 161-168.

Brown, C.C., Olander, H.J., Biberstein, E.L., Morse, S.M., 1986. Use of a toxoid vaccine to protect goats against intradermal challenge exposure to Corynebacterium pseudotuberculosis. Am. J. Vet. Res. 47, 1116-1119.

Brown, C.C., Olander, H.J., 1987. Caseous lymphadenitis of goats and sheep: a review. Vet. Bull. 57, 1-12.

Brown, C.C., Olander, H.J., Alves, F.C., 1987. Synergistic hemolysisinhibition titers associated with caseous lymphadenitis in a slaughterhouse survey of goats and sheep in northeastern Brazil. Can. J. Vet. Res. 51, 46-49.

Cameron, C.M., Minnaar, J.L., Engelbrecht, M.M., Purdom, M.R., 1972. Immune response of merino sheep to inactivated Corynebacterium pseudotuberculosis vaccine. Onderstepoort J. Vet. Res. $1,11-24$.

Carminati, R., Bahia, R., Moura-Costa, L.F., Paule, B.J.A., Vale, V.L., Regis, L., Freire, S.M., Nascimento, I., Schaer, R., Meyer, R., 2003. Determinação da sensibilidade e da especificidade de um teste de ELISA indireto para o diagnóstico de linfadenite caseosa em caprinos. R. Ci. Med. Biol. 2, 88-93.

Eggleton, D.G., Middleton, H.D., Doidge, D.G., Minty, D.W., 1991. Immunization against ovine caseous lymphadenitis: comparison with of Corynebacterium pseudotuberculosis vaccines with and without bacterial cells. Aust. Vet. J. 68, 317-319.

Ellis, T.M., Sutherland, S.S., Wilkinson, F.C., Mercy, A.R., Paton, M.W., 1987. The role of Corynebacterium pseudotuberculosis lung lesions in the transmission of this bacterium to other sheep. Austr. Vet. J. 64, 261-263.

Ellis, J.A., Hawk, D.A., Mills, K.W., Pratt, D.L., 1991. Antigen specificity of antibody responses to Corynebacterium pseudotuberculosis in naturally infected sheep with caseous lymphadenitis. Vet. Immunol. Immunopathol. 28, 289-301. 
Fontaine, M.C., Baird, G., Connor, K.M., Rudge, K., Sales, J., Donachie, W., 2006. Vaccination confers significant protection of sheep against infection with a virulent United Kingdom strain of Corynebacterium pseudotuberculosis. Vaccine 24, 5986-5996.

Hodgson, A.L., Bird, P., Nisbet, I.T., 1990. Cloning, nucleotide sequence, and expression in Escherichia coli of the phospholipase D gene from Corynebacterium pseudotuberculosis. J. Bacteriol. 172, 1256-1261.

Hodgson, A.L.M., Carter, K., Tachedjian, M., Krywult, J., Corner, L.A., McColl, M., Cameron, A., 1999. Efficacy of an ovine caseous lymphadenitis vaccine formulated using a genetically inactive form of the Corynebacterium pseudotuberculosis phospholipase D. Vaccine 17, 802-808.

Ioannou, X.P., Gomis, S.M., Karvonen, B., Hecker, R., Babiuk, L.A., Hurk, S.D.L., 2002. CpG-containing oligodeoxynucleotides, in combination with conventional adjuvants, enhance the magnitude and change the bias of the immune responses to a herpesvirus glycoprotein. Vaccine 21, 127-137.

Meyer, R., Regis, L., Vale, V., Paule, B., Carminati, R., Bahia, R., Moura-Costa, L., Schaer, R., Nascimento, I., Freire, S., 2005. In vitro IFN-gamma production by goat blood cells after stimulation with somatic and secreted Corynebacterium pseudotuberculosis antigens. Vet. Immunol. Immunopathol. 107, 249-254.

Mills, A.E., Mitchell, R.D., Lim, E.K., 1997. Corynebacterium pseudotuberculosis is a cause of human necrotising granulomatous lymphadenitis. Pathology 29, 231-233.

Moura-Costa, L.F., Paule, B.J.A., Freire, S.M., Nascimento, I., Schaer, R., Regis, L.F., Vale, V.L.C., Matos, D.P., Bahia, R.C., Carminati, R., Meyer, R., 2002. Meio sintético quimicamente definido para o cultivo de Corynebacterium pseudotuberculosis. Rev. Bras. Saúde Prod. Anim. 3, 1-8.

Nairn, M.E., Robertson, J.P., 1974. Corynebacterium pseudotuberculosis infection of sheep: role of skin and dipping fluids. Aust. Vet. J. 50, 537-542.

Nichani, A.K., Mena, A., Popowych, Y., Dent, D., Townsend, H.G.G., Mutwiri, G.K., Hecker, R., Babiuk, L.A., Griebel, P.J., 2004. In vivo immunostimulatory effects of $\mathrm{CpG}$ oligodeoxynucleotide in cattle and sheep. Vet. Immunol. Immunopathol. 98, 17-29.

Paule, B.J.A., Azevedo, V., Regis, L.F., Carminati, R., Bahia, R.C., Vale, V.L.C., Moura-Costa, L.F., Freire, S.M., Nascimento, I., Schaer, R., Goes, A.M., Meyer, R., 2003. Experimental Corynebacterium pseudotuberculosis primary infection in goats: kinetics of $\mathrm{IgG}$ and interferon production, $\mathrm{IgG}$ avidity and antigen recognition by Western Blotting. Vet. Immunol. Immunopathol. 96, 129-139.

Paule, B.J.A., Meyer, R., Moura-Costa, L.F., Bahia, R.C., Carminati, R., Regis, L.F., Vale, V.L.C., Freire, S.M., Nascimento, I., Schaer, R., Azevedo, V., 2004a. Three phase partitioning as an efficient method for extraction/concentration of immunoreactive excretedsecreted proteins of Corynebacterium pseudotuberculosis. Prot. Express. Purif. 34, 311-316.
Paule, B.J.A., Azevedo, V., Moura-Costa, L.F., Freire, S.M., Regis, L.F., Vale, V.L.C., Bahia, R.C., Carminati, R., Nascimento, I., Meyer, R., 2004b. SDS-PAGE and Western blot analysis of somatic and extracellular antigens of Corynebacterium pseudotuberculosis. R. Ci. Med. Biol. 3, 44-52.

Peel, M.M., Palmer, G.G., Stacpoole, A.M., Kerr, T.G., 1997. Human lymphadenitis due to Corynebacterium pseudotuberculosis: report of tem cases from Australia and review. Clin. Infect. Dis. 2, 185-191.

Pepin, M., Seow, H.F., Corner, L., Rothel, J.S., Hodgson, A.L.M., Wood, P.R., 1997. Cytokine gene expression in sheep following experimental infection with various strains of Corynebacterium pseudotuberculosis differing in virulence. Vet. Res. 28, 149-163.

Pepin, M., Sanchis, R., Paton, M., 1999. La lymphadénite caséeuse des ovins et des caprins. Point Vet. 30, 33-40.

Piontkowski, M.D., Shivvers, D.W., 1998. Evaluation of a commercial available vaccine against Corynebacterium pseudotuberculosis for use in sheep. J. Am. Vet. Med. Assoc. 212, 1765-1768.

Prescott, J.F., Menzies, P.I., Hwang, Y.T., 2002. An interferongamma assay for diagnosis of Corynebacterium pseudotuberculosis infection in adult sheep from a research flock. Vet. Microbiol. 88, 287297.

Rankin, R., Pontarollo, R., Ioannou, X., Krieg, A.M., Hecker, R., Babiuk, L.A., Hurk, S.D.L., 2001. CpG motif identification for veterinary and laboratory species demonstrates that sequence recognition is highly conserved. Antisense Nucleic Acid Drug Dev. 11, 333-340.

Simmons, C.P., Dunstan, S.J., Tachedjian, M., Krywult, J., Hodgson, A.L.M., Strugnell, R.A., 1998. Vaccine potential of attenuated mutants of Corynebacterium pseudotuberculosis in sheep. Infect. Immun. 66, 474-479.

Songer, J.G., Iandolo, J.J., Libby, S.J., Cuevas, W.A., 1990. Cloning and expression of the phospholipase D gene of Corynebacterium pseudotuberculosis in Escherichia coli. Infect. Immun. 1, 131-136.

Sting, R., Steng, G., Spengler, D., 1998. Serological studies on Corynebacterium pseudotuberculosis infections in goats using Enzyme-linked Immunosorbent Assay. J. Vet. Med. 45, 209-216.

Unanian, M.M., Feliciano-Silva, A.E.D., Pant, K.P., 1985. Abscesses and caseous lymphadenitis in goats in tropical semi-arid north-east Brazil. Trop. Anim. Health. Prod. 17, 57-62.

Walker, J., Jackson, H.J., Eggleton, D.G., Meeusen, E.N., Wilson, M.J., Brandon, M.R., 1994. Identification of a novel antigen from Corynebacterium pseudotuberculosis that protects sheep against caseous lymphadenitis. Infect. Immun. 62, 2562-2567.

Williamson, L.H., 2001. Caseous lymphadenitis in small ruminants. Vet. Clin. North. Am. Food Anim. Pract. 2, 359-371.

Wilson, M.J., Brandon, M.R., Walker, J., 1995. Molecular and biochemical characterization of a protective 40-Kilodalton antigen from Corynebacterium pseudotuberculosis. Infect. Immun. 63, 206-211. 